\section{Asociación VACTERL. Presentación de un caso en sesión anatomo- patológica y consideraciones generales}

\section{VACTERL Association. Presentation of an anatomoclinical case and general considerations.}

Ávila-Iglesias $\mathrm{MC}^{1}$, Rojas-Maruri $\mathrm{CM}^{2}$

\section{INTRODUCCIÓN}

La asociación VACTERL es un conjunto de malformaciones congénitas que tiene una baja prevalencia en la población general; ${ }^{1}$ sin embargo, está asociada a una elevada morbi-mortalidad. Se caracteriza por la presencia de diversas malformaciones en varias combinaciones, por lo que se cataloga como una asociación y no como síndrome con etiología única establecida.

La asociación VACTERL toma su nombre del acrónimo que corresponde a malformaciones congénitas que se presentan característicamente: malformaciones Vertebrales, atresia Anal, anomalías Cardiovasculares, fistula Traqueoesofágica, atresia Esofágica, malformaciones Renales y displasia de las extremidades (Limbs), fundamentalmente en el hueso radial. ${ }^{2}$

El primer reporte de la asociación VATER fue realizado por Quan y Smith en 1972, donde la letra R correspondía a displasia ra-
${ }^{1}$ Médico Adscrito al Departamento de Neonatología. ${ }^{2}$ Médico adscrito al Departamento de Anatomía Patológica.

Instituto Nacional de Pediatría, Ciudad de México.

Recibido: 28 de junio del 2017

Aceptado: 1 de agosto del 2017

Correspondencia

María del Carmen Ávila-Iglesias

mcavilai@yahoo.com

Este artículo debe citarse como

Ávila-Iglesias MC, Rojas-Maruri CM. Asociación VACTERL. Presentación de un caso en sesión anatomo-patológica y consideraciones generales. Acta Pediatr Mex. 2017;38(5):330-336. 
dial. Posteriormente, Temtamy y Miller usaron el acrónimo VATERS, en el cual la $\mathrm{V}$ representaba malformaciones vertebrales y defectos en el septum ventricular y la $\mathbf{S}$ una sola arteria umbilical. Finalmente, se adopta el acrónimo VACTERL en 1975 (C: defectos cardiacos y L: anomalías en las extremidades) que continúa en uso hasta este momento. Su incidencia es de 1 por cada 10,000 a 40,000 recién nacidos vivos y tiene predilección por el sexo masculino con una relación $2.6: 1 .^{2,3}$

\section{ETIOLOGÍA}

La etiología es desconocida y probablemente multifactorial, por lo que no puede catalogarse como un síndrome específico, además sus componentes son variables. Se ha sugerido que la exposición a estrógenos, progestágenos o ambos durante el primer trimestre del embarazo puede ser una causante de esta entidad. Su herencia es de carácter esporádico, aunque la aparición de varios casos en una familia sugiere una herencia de carácter autosómico, así como los casos de VACTERL con hidrocefalia, que siguen un patrón de herencia autosómico recesivo. ${ }^{1,4}$

\section{DIAGNÓSTICO}

Para poder establecer el diagnóstico de asociación VACTERL se requiere de al menos tres de los siete criterios que se muestran en el Cuadro $1 .^{5}$ Para poder mejorar el pronóstico de estos pacientes es indispensable realizar un diagnóstico ultrasonográfico prenatal oportuno a partir de la semana 18 de gestación, ya que a esta edad gestacional se permite identificar la mayoría de las alteraciones estructurales del feto. El ultrasonido estructural debe ser realizado por personal capacitado para incrementar la posibilidad de detectar malformaciones sutiles. La sensibilidad y la especificidad del ultrasonido en la detección de esta asociación es de 84 y 99.9\%, respectiva- mente. ${ }^{5}$ La detección de anomalías congénitas de cualquier tipo en el ultrasonido obstétrico en el segundo trimestre de embarazo es fundamental para el diagnóstico de esta patología. Entre los exámenes que hay que realizar al nacimiento se encuentran: ultrasonido renal, ecocardiograma y radiografía de columna y huesos largos. El diagnóstico diferencial incluye la asociación de VACTERL-H (síndrome de Briard-Evans), que es una asociación VACTER con hidrocefalia que se piensa tiene una herencia autosómica recesiva y trastorno distinto del VACTER. Este síndrome tiene un peor pronóstico y se ha asociado con cuadros graves de anemia de Fanconi. En el diagnóstico diferencial de la asociación VACTERL también se incluyen: cromosomopatías como la trisomía 18 y 13, el síndrome de Klippel Feil y Goldenhar, la trombocitopenia con radio ausente, la anemia de Fanconi, los síndromes de Robert, de Holt-Oram y el de Nager; el síndrome de regresión caudal. ${ }^{3,4}$

\section{TRATAMIENTO Y PRONÓSTICO}

La morbi-mortalidad y el pronóstico a largo plazo dependen directamente de la oportuna corrección quirúrgica inicial de los defectos que pongan el riesgo la vida del recién nacido, tales como atresia esofágica y atresia anal. Posteriormente se realizarán las siguientes correcciones quirúrgicas no urgentes que el paciente necesite, garantizando el seguimiento y rehabilitación mediante terapias físicas y del lenguaje. Debido a que el neurodesarrollo de estos pacientes es normal, no se deben escatimar esfuerzos tanto quirúrgicos como de rehabilitación. La falla renal es una complicación grave y común, por tal motivo el diagnóstico prenatal es fundamental para mejorar el pronóstico de estos pacientes. La morbi-mortalidad de estos pacientes depende de las complicaciones antes mencionadas, incluyendo la falla renal y las complicaciones propias del postquirúrgico. ${ }^{5}$ 
Cuadro 1. Criterios diagnósticos de la Asociación VACTERL

\begin{tabular}{|ll}
\hline Defectos vertebrales & $\begin{array}{l}\text { Vértebras hipoplásicas o hemivértebras escoliosis } \\
\text { Xifosis lordosis }\end{array}$ \\
\hline Malformaciones anales & Estenosis anal \\
& Otras malformaciones ano-rectales \\
\hline Cardiopatías congénitas & Comunicación interauricular \\
& Comunicación interventricular \\
\hline Alteraciones traqueo-esofágicas & Fístula traqueo-esofágica \\
& Atresia de esófago \\
\hline Malformaciones renales & $\begin{array}{l}\text { Anomalías ureterales } \\
\text { Hidronefrosis }\end{array}$ \\
& Agenesia renal \\
\hline Alteraciones en las & Malformaciones en las extremidades inferiores \\
extremidades (limbs) & Ausencia de radio, tibia, peroné \\
Ausencia de dedos \\
Polidactilia \\
Sindactilia \\
Malformación del pulgar
\end{tabular}

\section{PRESENTACIÓN DEL CASO CLÍNICO}

Recién nacido de sexo indeterminado producto de la gesta 2, cesárea 1 de madre de 23 años. Control prenatal desde el diagnóstico de embarazo, con un total de 15 consultas y 15 ultrasonidos con detección de múltiples malformaciones, no especificadas en nota de referencia. Se mencionó en dicha nota que por mal pronóstico se propuso aborto terapéutico en dos unidades hospitalarias, no aceptado por la madre. Acudió a revisión a clínica particular donde encontraron frecuencia cardiaca fetal de 60 latidos por minuto y probable multiquistosis renal bilateral; por pérdida de bienestar fetal, oligohidramnios grave, se decidió interrupción del embarazo vía abdominal, bajo anestesia epidural. Se obtuvo producto único vivo, con peso de 1600 gramos y talla de $47 \mathrm{~cm}$. Se otorgaron 35 semanas de gestación por Capurro. Silverman 2 y Apgar 6-7. Con frecuencia cardiaca de 80 latidos por minuto, por lo que se requirió de un ciclo de ventilación con presión positiva intermitente con bolsa y máscara. Se mantuvo con ventilación con mascarilla de no reinhalación para adultos.
A su ingreso a Urgencias del hospital de referencia se encontró al paciente hipoactivo e hiporreactivo con cianosis central y dificultad respiratoria con Silverman Andersen de 2-3. Por datos de dificultad respiratoria y síndrome dismórfico se refirió a hospital de tercer nivel para su abordaje diagnóstico y terapéutico. Tuvo un traslado de 2 horas de duración sin registro de tensión arterial ni uresis y donde presentó paro cardiorrespiratorio de 2 minutos de duración. Se recibió en nuestra institución con palidez generalizada, frecuencia cardíaca de 100 latidos por minuto, ventilado con bolsa-válvula-mascarilla de adulto con saturación de 40\%, glucometría capilar no cuantificable (i.e. "alta") y temperatura de 35 grados centígrados, por lo que se intubó y se ingresó a la Unidad de Cuidados Intensivos Neonatales.

Exploración física: paciente con palidez generalizada de tegumentos, hipotérmico, cráneo se apreció microcéfalo, con perímetro cefálico de $29 \mathrm{~cm}$, fontanela anterior puntiforme, fontanela posterior cerrada, normotenso, con adecuada implantación de cabello e implantación baja de pabellones auriculares, hipotelorismo ocu- 
lar, puente nasal aplanado, narinas permeables sin secreciones, retrognatia, paladar íntegro, cuello sin adenomegalias, tórax simétrico con hipoventilación en hemitórax derecho, precordio con ruidos cardiacos rítmicos, de adecuada intensidad, disminuidos en frecuencia, no se auscultaron soplos, abdomen blando depresible, sin visceromegalias, se observó cordón umbilical ligado, no se lograron identificar vasos umbilicales. Genitales con falo de $0.8 \mathrm{~cm}$, meato central no se palparon gónadas, extremidades hipotróficas, con pulsos pedios débiles en ambas extremidades inferiores, se palparon pulsos braquiales e inguinales. Se observó malformación anorrectal sin fístula evidente además de deformidad en varo de ambas extremidades inferiores.

Al Ilegar a la Unidad de Cuidados Intensivos Neonatales persistió sin detección de tensión arterial, a pesar de infusiones de dobutamina y adrenalina, manteniendo 55\% de saturación de oxígeno que no mejoró a pesar aumento en los parámetros ventilatorios. Presentó paro cardiorrespiratorio de 3 minutos que revirtió con maniobras avanzadas de reanimación. Simultáneamente, se corroboró intubación correcta ante la persistencia de oximetría de pulso menor a $60 \%$ y aunado a hipoventilación en hemitórax derecho se colocó minisello pleural, recuperando saturación al 70\%. Posteriormente presentó segundo paro cardiorrespiratorio irreversible a maniobras avanzadas.

\section{HALLAZGOS ANATOMOPATOLÓGICOS}

Se realizó estudio de necropsia a paciente con edad aparente igual a la cronológica, en el aspecto externo con microcefalia, implantación baja de pabellones auriculares, hipotelorismo, puente nasal aplanado, retrognatia, presencia de ano imperforado, pie equinovaro bilateral y testículos no descendidos.

Al estudio de microscopia se encontraron en el pulmón membranas hialinas (Figura 1) que



Figura 1. La imagen microscópica mostró abundante material proteináceo en la luz de los alvéolos.

generaron hipoxia importante que ocasionó el deceso del paciente. Adicionalmente, al momento de la evisceración, se observó displasia renal quística izquierda con agenesia renal derecha, malformación anorrectal con fístula recto-vesical (Figura 2), hemivértebras torácicas y pie equino varo bilateral, con lo que se integró asociación VACTERL. Cerebro, corazón, esófago y tráquea no mostraron patología alguna al ser explorados.

Aunque la causa del deceso del paciente fue la hipoxia grave que presentó secundaria a la enfermedad de membrana hialina, la autopsia nos ayudo a encontrar alteraciones anatómicas adicionales a las encontradas externamente, lo cual nos permitió integrar el diagnóstico de asociación VACTERL. Con lo anterior, queda una vez más demostrada la importancia de los estudios de necropsia.

Los diagnósticos finales del estudio fueron los siguientes:

I. Enfermedad de membrana hialina con datos histológicos de inmadurez pulmonar.
A. Miopatía hipóxica. 


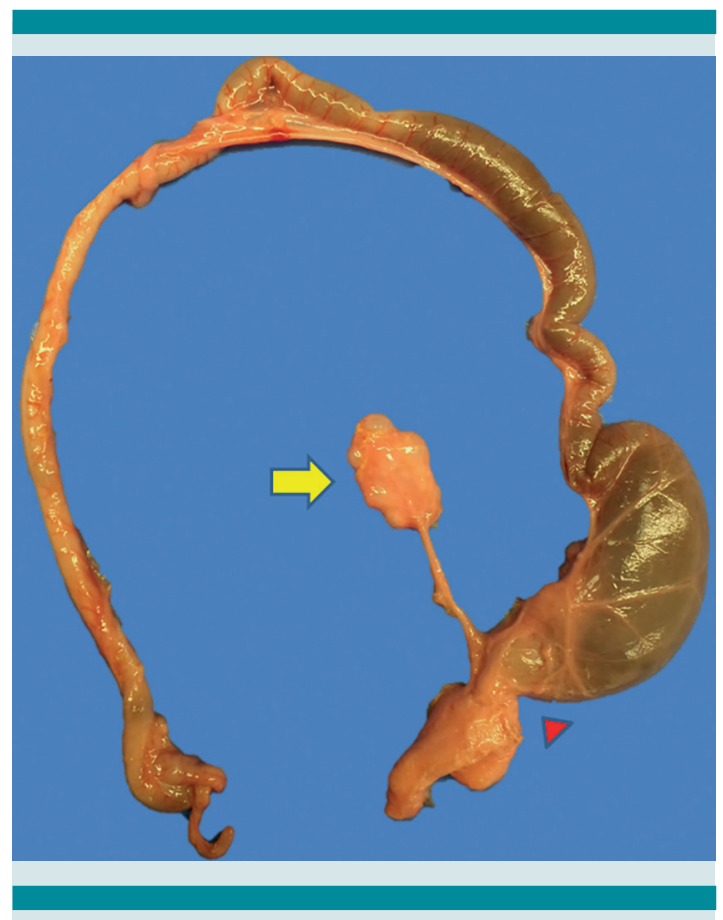

Figura 1. Foto macroscópica que muestra segmento de colon descendente dilatado, con fístula hacia vejiga (punta de flecha roja). También se observa riñón de lado izquierdo disminuido de tamaño (1.1 vs. 17 g) y de aspecto multiquístico.

II. Asociación VACTERL:

A. Displasia renal quística izquierda con agenesia renal derecha.

B. Malformación anorrectal con fístula recto-vesical.

C. Hemivértebra torácica.

D. Extremidades cortas e hipotróficas (exploración física).

E. Pie equino varo bilateral.

III. Síndrome dismórfico (historia clínica)

A. Microcefalia.

B. Implantación baja de pabellones auriculares.
C. Hipotelorismo.
D. Puente nasal aplanado.
E. Retrognatia.

IV. Esofagitis ulcerada con hemorragia focal de la submucosa.

Los puntos clave que debemos recordar respecto a las asociaciones de acuerdo a Enid GilbertBarness y sus colaboradores son: ${ }^{6}$

1. Una asociación es una entidad biológica que puede presentarse en un niño o feto.

2. Las asociaciones pueden solaparse entre sí y confundirse con entidades similares, por lo que el diagnóstico en ocasiones resulta difícil.

3. Las asociaciones pueden tener una alta incidencia (1 en 820 nacimientos).

4. Las asociaciones suelen ser casos esporádicos con una muy baja recurrencia empírica.

5. Las asociaciones parecen ser no específicas.

6. Las asociaciones parecen representar múltiples "hits" involucrando primordios vulnerables.

7. La mayoría de las asociaciones afectan estructuras de la línea media.

8. Sustancias teratógenas pueden estar involucradas en la blastogénesis ocasionando alteraciones en el desarrollo.

De acuerdo a la evaluación realizada por el servicio de genética, el paciente contaba con 
los criterios mínimos necesarios para realizar el diagnóstico de asociación VACTERL. Debido al poco tiempo que el paciente permaneció en el hospital no fue posible realizar estudios de imagen que confirmaran las alteraciones descritas a la exploración clínica, sin embargo, pensamos que lo observado es suficiente para realizar el diagnóstico de asociación VACTERL.

\section{DISCUSIÓN}

La asociación de VACTERL es poco frecuente en la población general, con una incidencia reportada de 1 por cada 10,000 a 40,000 recién nacidos vivos; ${ }^{2,3}$ sin embargo, es una patología con una alta morbi-mortalidad asociada, por lo cual es fundamental el diagnóstico de sospecha o certero desde los controles prenatales.

De acuerdo con la Norma Oficial Mexicana 007-SSA2 (Atención de la mujer durante el embarazo, parto y puerperio y del recién nacido ${ }^{7}$ y la Norma Oficial Mexicana 034-SSA2 (Para la prevención y control de los defectos al naci-

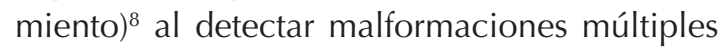
durante los ultrasonidos obstétricos se debió haber referido oportunamente a la madre a un tercer nivel de atención. Esto, con el fin de continuar el control del embarazo y asegurar una adecuada atención perinatal al nacimiento, tanto para la madre como para el producto. En el caso de confirmarse malformaciones fetales incompatibles con la vida postnatal podría considerarse la interrupción del embarazo, después de una evaluación multidisciplinaria.

En este caso se trató de un paciente que aparentemente contaba con diagnóstico prenatal certero, ya que se mencionó la realización de varios ultrasonidos que detectaron malformaciones fetales múltiples; sin embargo, desconocemos si los ultrasonidos fueron realizados por personal capacitado y cuales eran las malformaciones encontradas. A pesar de esto, la madre no fue referida oportunamente a un tercer nivel de atención. Además, posterior al nacimiento, las condiciones de traslado fueron inadecuadas. Esto condicionó que el paciente llegara en estado de choque, con riesgo de sepsis y requiriera soporte ventilatorio desde su ingreso. Existen lineamientos internacionales para la adecuada reanimación al nacimiento y para el correcto traslado de pacientes neonatos que optimizan la supervivencia. A escala mundial se deben seguir, de manera estandarizada, los lineamientos del Programa de Reanimación Neonatal 7a edición (Neonatal Resuscitation Program o NRP) ${ }^{9}$ para la atención en cada nacimiento en un medio hospitalario. El programa STABLE (Sugar-Temperature-Airway-Blood pressure-Lab work and Emotional support program) ${ }^{10}$ tiene como objetivos específicos la estabilización y el manejo adecuado del neonato antes y durante el traslado. El programa ACoRN (Acute Care of at Risk Newborns) ${ }^{11}$ está enfocado en la evaluación y manejo del neonato en estado crítico. La atención de neonatos con malformaciones congénitas es compleja y requiere la mayoría de las veces de atención especializada. El mejor transporte que podemos ofrecerle a un neonato con malformación congénita es el útero de su madre y la única forma de ofrecer esta opción es con un diagnóstico prenatal oportuno. Además, se debe asegurar que el traslado del paciente posterior al nacimiento sea realizado por personal capacitado.

A pesar de ser un prematuro limítrofe de 35 semanas de gestación, en los cuales la enfermedad por membranas hialinas no es frecuente, en este paciente el consumo de surfactante fue secundario a compromiso hemodinámico condicionado por las malas condiciones de traslado, y no como consecuencia de la prematurez. El traslado inadecuado provocó también un compromiso hemodinámico importante, que aunado al consumo de surfactante condicionaron la muerte del paciente. A pesar de que, aparentemente 
tenía diagnóstico prenatal de malformaciones incompatibles con la vida, las cuales no fueron referidas en la hoja de traslado; si el paciente hubiera sido trasladado en forma adecuada a tercer nivel podría haber sobrevivido hasta completar estudios de extensión.

Como conclusión, la asociación VACTERL es un conjunto de malformaciones congénitas, que tiene baja incidencia, pero una morbi-mortalidad elevada, por lo que es fundamental un diagnóstico prenatal adecuado, y una referencia oportuna a tercer nivel de atención. Es de suma importancia recalcar que el mejor traslado es en el vientre materno, con una referencia oportuna de la madre a tercer nivel de atención. En caso de que el diagnóstico se sospeche en el período postnatal es fundamental que el traslado se realice de acuerdo a los lineamientos internacionales y por personal capacitado.

\section{REFERENCIAS}

1. Bacino C. Birth defects: Approach to evaluation. www. uptodate.com @2017 UpToDate.
2. Solomon BD, Baker LA., et al. An Approach to the Identification of Anomalies and Etiologies in Neonates with Identified or Suspected VACTERL (Vertebral Defects, Anal Atresia, Tracheo-Esophageal Fistula with Esophageal Atresia, Cardiac Anomalies, Renal Anomalies, and Limb Anomalies) Association.J Pediatr. 2014;164(3):451-7.

3. Solomon B. VACTERL/VATER Association. Orphanet Journal of Rare Diseases 2011;6:56.

4. Solomon BD, Bear KA, et al. Clinical Geneticists' Views of VACTERL/VATER Association. Am J Med Genet A. 2012;158A(12):3087-3100.

5. Martinez $\mathrm{S}$, et al. A case with multiple congenital malformations: VACTERL association. Med UNAB. 2011;14:132-7.

6. Enid Gilbert-Barness, AO, MD, FRCPA, FRCPath, DSci(hc) and cols., Potter's Pathology of the Fetus, Infant and Child, 2nd Edition.2007;1:71-2.

7. Norma Oficial Mexicana NOM-007-SSA2-1993, Atención de la mujer durante el embarazo, parto y puerperio y del recién nacido. Criterios y procedimientos para la prestación del servicio.

8. Norma Oficial Mexicana NOM-034-SSA2-2002, Para la prevención y control de los defectos al nacimiento.

9. American Academy of Pediatrics/American Heart Association. Textbook of Neonatal Resucitation 7th Edition. 2016.

10. Karlsen K. The S.T.A.B.L.E. Program 6th Edition. 2012.

11. Canadian Pediatric Society. ACoRN: Acute Care of At-Risk Newborns. 2012 Update. 